\title{
Synthesis and antibacterial activity of sulfonamide derivatives at C-8 alkyl chain of anacardic acid mixture isolated from a natural product cashew nut shell liquid (CNSL)
}

\author{
N SUBHAKARA REDDY ${ }^{\mathrm{a}, \mathrm{b}}$, A SRINIVAS RAO ${ }^{\mathrm{a}}$, M ADHARVANA CHARI $^{\mathrm{c}, \mathrm{d}, *}$, \\ V RAVI KUMAR $^{\mathrm{a}}$, V JYOTHY ${ }^{\mathrm{c}}$ and V HIMABINDU ${ }^{\mathrm{b}}$ \\ ${ }^{a}$ Medicinal chemistry Laboratory, GVK Biosciences Pvt Ltd, Plot No 5C, IDA, Uppal Road, \\ Hyderabad 500 039, India \\ ${ }^{\mathrm{b}}$ Centre for Environment, Institute of Science and Technology, JNT University, Kukatpally, \\ Hyderabad 500 085, India \\ ${ }^{c}$ Dr. MACS Bio-Pharma Pvt. Ltd., Plot-32/A,Westren Hills, Kukatpally, Hyderabad 500 085, India \\ ${ }^{\mathrm{d}}$ Department of Complexity Science and Engineering, School of Frontier Sciences, 5-1-5, Kashiwanoha, \\ Kashiwa, University of Tokyo, Chiba 277 8561, Japan \\ e-mail:drmac_s@yahoo.com
}

MS received 20 August 2011; revised 11 December 2011; accepted 26 December 2011

\begin{abstract}
Synthesis and antibacterial activity of some novel biologically active sulfonamide derivatives at C-8 alkyl chain of anacardic acid (7a-7l), prepared from commercially available anacardic acid mixture (1a-d). These compounds were tested for Gram positive and Gram negative bacterial cultures; most of the compounds showed higher antibacterial activity compared with standard drug ampicillin.
\end{abstract}

Keywords. Synthesis; sulfonamide derivatives; anacardic acid; antibacterial activity.

\section{Introduction}

In continuation of our ongoing investigation in the search of new bioactive compounds using non-isoprenoid phenolic lipids from Anacardium occidentale as starting material, we describe here the synthesis and biological activity of some novel sulfonamide derivatives at C-8 alkyl chain of anacardic acid mixture isolated from a natural product Cashew Nut Shell Liquid (CNSL). Anacardic acid mixture (1a-d) isolated from a natural product CNSL which is a by-product of cashew nut industry and these are salicylic acid derivatives with a non-isoprenoid alk(en)yl side chain. ${ }^{1}$ Anacardic acid and its derivatives exhibit biological activities like antimicrobial activity 2,3 and soybean lipoxygenase-1 inhibitory activity ${ }^{4,5}$ Reddy et al. reported the synthesis of benzamide derivatives of anacardic acid ${ }^{6}$ sildenafil analogues ${ }^{7}$ dihydropyridine analogues ${ }^{8}$ as calcium channel blockers, isonicotinoylhydrazones for antimycobacterial activity ${ }^{9}$ starting from anacardic acid. Kubo et al. reported the separation of anacardic acid into monoene (15:1), diene (15:2) and triene (15:3) by preparative high pressure liquid chromatography and

*For correspondence tested against cancer cells, and found to show moderate cytotoxic activity on BT-20 breast and HeLa epithelioid cervix carcinoma cells. ${ }^{10}$ Recently, a few anacardic acid derivatives exhibited various activities like affect of the structure of the enzyme, ${ }^{11}$ anacardic acid is a specific activator of kinase activity of Aurora Kinase $\mathrm{A},{ }^{12}$ suppresses expression of nuclear factor-kB regulated gene products leading to potentiation of apoptosis ${ }^{13}$ inhibitor of the HAT activity of recombinant Plasmodium falciparum $\mathrm{GCN} 5^{14}$ and as modulators of histone acetyltransferases. ${ }^{15}$

The emergence of drug-resistant strains in clinical applications ${ }^{16-18}$ especially to gram positive bacteria ${ }^{19,20}$ has created a problem of global proportions. ${ }^{21,22}$ This phenomenon has led in creating novel antibacterial agents distinct from the existing classes of compounds. Anacardic acid (pentadecyl salicylic acid) is a phenolic constituent present in CNSL; (Anacardium occidentale L.) and exhibits antimicrobial properties ${ }^{2,3}$ which have led to the preparation of various analogues. ${ }^{23,24}$ Synthesis of lasiodiplodin from the nonisoprenoid phenolic lipids of CNSL as well as the salicylate macrolactone and other derivatives were reported by Santos et al. ${ }^{25}$ The interesting chemical characteristics of anacardic acid, such as the presence of a double 
bond at the 8-position of the long-chain in the monoene, diene and triene components and a convenient aromatic system led us to search for a strategy to convert these materials into useful products. In the present work, we wish to report the synthesis of novel cell permeable sulfonamide compounds from abundantly and cheaply available anacardic acid which was a major constituent of CNSL natural source to evaluate their biological activity by various antibacterial strains. This report describes the synthesis, spectroscopic identification and antibacterial activity of some novel sulfonamide derivatives at C-8 alkyl chain of anacardic acid mixture against Escherichia coli, Pseudomonas aeruginosa, Staphylococcus aureus and Streptococcus pyogenes bacterial strains.

\section{Experimental}

\subsection{General consideration}

All chemicals and solvents were obtained from Aldrich and Spectrochem, India and used without further purification. Column chromatographic separations were carried out on silica gel 60-120 mesh size and eluting with a gradient of hexane: ethyl acetate. Analytical thin layer chromatography was performed on pre-coated Merck silica gel (60F254/0.2 mm) plates using UV light, 5\% ethanolic phosphomolybdic acid or iodine vapours to visualize the spots. Melting points were determined in open glass capillaries on a Mel-temp apparatus and are uncorrected. The IR spectra were recorded on a Thermo Nicolet IR 200 FT-IR spectrometer as $\mathrm{KBr}$ pellets and the wave numbers were given in $\mathrm{cm}^{-1}$. The ${ }^{1} \mathrm{H}$ and ${ }^{13} \mathrm{C}$ NMR spectra of samples were recorded on a Varian EM-360, NMR spectrometer using TMS as an internal standard in $\mathrm{CDCl}_{3}$. The mass spectra were recorded on Jeol JMS-D 300 and Finnigan Mat b at $70 \mathrm{eV}$ with an emission current of $100 \mu \mathrm{A}$. The oxidative cleavages were performed with a Welsbach T-408 ozonizator and the catalytic hydrogenations in a Parr apparatus.

2.1a General procedure-Isolation of anacardic acids (1): The shells $(500 \mathrm{~g})$ of cashew nuts from Anacardiumoccidentale (Ceará, Brazil) were extracted in a Soxhletextractor with commercial $95 \%$ ethanol $(2.0 \mathrm{~L})$ during $6 \mathrm{~h}$, yielding a crude extract (CNSL, $157 \mathrm{~g}, 31 \%$ by weight). Anacardic acids (1) were removed in $61 \%$ from CNSL ( $15.25 \mathrm{~g}$ ) either by precipitation with lead nitrate or calcium hydroxide according to protocols described in the literature. ${ }^{26}$ The spectral properties were identical to those reported in the literaure. ${ }^{26 a}$ 2.1b Synthesis of methyl anacardate methyl ethers ene mixture (2): To a solution of compound $\mathbf{1}$ $(65 \mathrm{~g}, 186.78 \mathrm{mmol})$ in acetone was added $\mathrm{K}_{2} \mathrm{CO}_{3}$ $(103.1 \mathrm{~g}, 747.12 \mathrm{mmol})$, and di methyl sulfate $(44.3 \mathrm{~mL}$, $466.95 \mathrm{mmol})$. The contents were heated at $65^{\circ} \mathrm{C}$ for $5 \mathrm{~h}$. Reaction mixture was cooled to room temperature, filtered and washed with ethyl acetate. Filtrate was distilled off, crude compound was re-dissolved in ethyl acetate $(300 \mathrm{~mL})$. Organic layer was washed with water, brine solution and dried over anhydrous sodium sulphate and distilled off ethyl acetate. Crude compound was purified by $60-120$ silica pet ether pack column compound was eluted with 5\% ethyl acetate: pet ether to get compound 2, Yield: $58 \mathrm{~g}$, nature of compound: light yellow liquid.

2.1c 2-(8-Hydroxy-octyl)-6-methoxy-benzoic acid methyl ester (3): A solution of $2(15 \mathrm{~g}, 40.540 \mathrm{mmol})$ in dichloro methane: methanol $(1: 1,500 \mathrm{~mL})$ was cooled to $-78^{\circ} \mathrm{C}$ and treated with a stream of $\mathrm{O}_{3}$ gas until the completion of starting material. The reaction mixture was treated with the stream of $\mathrm{O}_{2}$ at $-78^{\circ} \mathrm{C}$ to remove excess amount of $\mathrm{O}_{3}$. To the reaction mixture was added to a solution of dimethyl sulfide (catalytic qty) at $-78^{\circ} \mathrm{C}$, and the mixture was allowed to stir at room temperature for $2 \mathrm{~h}$. The reaction mixture was again cooled to $-15^{\circ} \mathrm{C}$, and was added sodium borohydride $(9.97 \mathrm{~g}, 0.26 \mathrm{~mol})$ portion-wise over a period of $45 \mathrm{~min}$; the reaction mixture was allowed to reach room temperature and stirred for $18 \mathrm{~h}$. To the reaction mixture, cold water was added $(400 \mathrm{~mL})$ and the aqueous layer was extracted with dichloromethane $(2 \times 200 \mathrm{~mL})$. The combined organic layer was washed with brine solution $(150 \mathrm{~mL})$ dried over anhydrous sodium sulphate and concentrated. The residue was purified by neutral alumina column chromatography (eluted with $20 \%$ ethyl acetate: pet ether) to afford $\mathbf{3}$ as yellow liquid; Yield: $7.1 \mathrm{~g}$ (59.5\%); IR (neat): $\mathrm{n}_{\max }$ 3401, 2930, 1728, 1586, 1467, 1268, 1110, 1071, 954, $749 \mathrm{~cm}^{-1}$; ${ }^{1} \mathrm{H}-\mathrm{NMR}\left(400 \mathrm{MHz}, \mathrm{CDCl}_{3}\right): \delta 1.31$ (bs, 8H), $1.53-$ $1.59(\mathrm{~m}, 4 \mathrm{H}), 2.53(\mathrm{t}, 2 \mathrm{H}, J=8.0 \mathrm{~Hz}), 3.63(\mathrm{t}, 2 \mathrm{H}, J=$ $6.8 \mathrm{~Hz}), 3.81(\mathrm{~s}, 3 \mathrm{H}), 3.90(\mathrm{~s}, 3 \mathrm{H}), 6.75(\mathrm{~d}, 1 \mathrm{H}, J=$ $8.4 \mathrm{~Hz}), 6.82(\mathrm{~d}, 1 \mathrm{H}, J=7.6 \mathrm{~Hz}), 7.26-7.28(\mathrm{~m}, 1 \mathrm{H})$; ${ }^{13} \mathrm{C}$ NMR $\left(100 \mathrm{MHz}, \mathrm{CDCl}_{3}\right), \delta: 25.58,29.16,29.23$, $29.63,31.00,32.62,33.35,52.10,55.73,62.81,108.23$, 121.37, 123.26, 130.18, 141.17, 156.10, 168.93; EI MS: m/z (Relative abundance \%) $295\left(\mathrm{M}^{+}, 100\right)$.

2.1d Synthesis of 2-(8-bromo-octyl)-6-methoxy-benzoic acid methyl ester (4): A solution of compound $\mathbf{3}(15 \mathrm{~g}$, $51.02 \mathrm{mmol})$ in dichloro methane $(150 \mathrm{~mL})$ was added to dry pyridine $(42 \mathrm{~mL}, 510.2 \mathrm{mmol})$ and tri phenyl 
phosphene $(22.73 \mathrm{~g}, 86.734 \mathrm{mmol})$ at $0^{\circ} \mathrm{C}$. Carbon tetra bromide $(25.4 \mathrm{~g}, 76.53 \mathrm{mmol})$ was added portion-wise over a period of $15 \mathrm{~min}$. The mixture was slowly brought it to room temperature and stirred for $6 \mathrm{~h}$. Reaction mixture was diluted with DCM $(100 \mathrm{~mL})$ washed with $2 \mathrm{~N} \mathrm{HCl}(2 \times 150 \mathrm{~mL})$, water $(200 \mathrm{~mL})$, brine solution $(175 \mathrm{~mL})$, dried over anhydrous $\mathrm{Na}_{2} \mathrm{SO}_{4}$, filtered and evaporated under vacuum, Crude compound was purified by column chromatography using $10 \%$ ethyl acetate in pet ether to get pure compound $\mathbf{4}$ as yellow liquid; Yield: $16.5 \mathrm{~g}$ (90.8\%); IR (DCM film): $v_{\max } 3071,3002,2931,2854,1732,1588,1464,1437$, 1268, 1109, 1072, 960, $749 \mathrm{~cm}^{-1} ;{ }^{1} \mathrm{H}$ NMR $400 \mathrm{MHz}$, $\left.\mathrm{CDCl}_{3}\right): \delta 1.29-1.42(\mathrm{~m}, 8 \mathrm{H}), 1.52-1.59(\mathrm{~m}, 2 \mathrm{H}), 1.80$ $1.87(\mathrm{~m}, 2 \mathrm{H}), 2.53(\mathrm{t}, 2 \mathrm{H}, J=8.0 \mathrm{~Hz}), 3.40(\mathrm{t}, 2 \mathrm{H}$, $J=7.2 \mathrm{~Hz}), 3.82(\mathrm{~s}, 3 \mathrm{H}), 3.90(\mathrm{~s}, 3 \mathrm{H}), 6.76(\mathrm{~d}, 1 \mathrm{H}$, $J=8.4 \mathrm{~Hz}), 6.81(\mathrm{~d}, 1 \mathrm{H}, J=7.6 \mathrm{~Hz}), 7.24-7.28$ $(\mathrm{m}, 1 \mathrm{H}) \mathrm{ppm} ;{ }^{13} \mathrm{C} \mathrm{NMR}\left(100 \mathrm{MHz}, \mathrm{CDCl}_{3}\right): \delta 28.05$, 28.56, 29.12, 29.26, 31.02, 32.72, 33.36, 34.02, 52.13, 55.78, 108.27, 121.39, 123.32, 130.20, 141.14, 156.14, 168.90 ppm; ESIMS (m/z): $357(\mathrm{M}+\mathrm{H})^{+} .359$ (bromo).

2.1e Synthesis of 2-(8-aza-octyl)-6-methoxy-benzoic acid methyl ester (5): A solution of compound 4 $(2.0 \mathrm{~g}, 5.617 \mathrm{mmol})$ in DMF $(10 \mathrm{~mL})$ was added to sodium azide ( $548 \mathrm{mg}, 8.426 \mathrm{mmol}$ ). The mixture was heated at $100^{\circ} \mathrm{C}$ for $3 \mathrm{~h}$, reaction mixture was poured into cool water $(70 \mathrm{~mL})$ and extracted with diethyl ether $(2 \times 40 \mathrm{~mL})$, the organic layer was washed with water $(50 \mathrm{~mL})$, brine solution $(30 \mathrm{~mL})$, dried over anhydrous $\mathrm{Na}_{2} \mathrm{SO}_{4}$, filtered and evaporated under vacuum to obtain compound 5 as yellow liquid; Yield:1.5 g (83.7\%); IR (DCM film): $v_{\max } 3087,3002,2932,2856,2095,1731$, 1588, 1464, 1266, 1109, 1071, $753 \mathrm{~cm}^{-1} ;{ }^{1} \mathrm{H}$ NMR $\left(400 \mathrm{MHz}, \mathrm{CDCl}_{3}\right): \delta 1.30$ (bs, 8H), 1.59 (brs, 4H), 2.53 $(\mathrm{t}, 2 \mathrm{H}, J=7.6 \mathrm{~Hz}), 3.25(\mathrm{t}, 2 \mathrm{H}, J=7.2 \mathrm{~Hz}), 3.82(\mathrm{~s}$, $3 \mathrm{H}), 3.91(\mathrm{~s}, 3 \mathrm{H}), 6.76(\mathrm{~d}, 1 \mathrm{H}, J=8.4 \mathrm{~Hz}), 6.82(\mathrm{~d}$, $1 \mathrm{H}, J=8.0 \mathrm{~Hz}), 7.25-7.29(\mathrm{~m}, 1 \mathrm{H}) \mathrm{ppm} ; \operatorname{ESIMS}(\mathrm{m} / \mathrm{z})$ : $320(\mathrm{M}+\mathrm{H})^{+}$.

2.1f Synthesis of 2-(8-Amino-octyl)-6-methoxy-benzoic acid methyl ester (6): A solution of compound 5 $(2.0 \mathrm{~g}, \mathrm{mmol})$ in ethanol $(30 \mathrm{~mL})$ was taken into a $500 \mathrm{~mL}$ Parr-hydrogenation vessel and added a suspension of $10 \% \mathrm{Pd} / \mathrm{C}(220 \mathrm{mg}, 10 \%)$ in $20 \mathrm{~mL}$ of ethanol under argon atmosphere and applied $\mathrm{H}_{2-}$ pressure $(60 \mathrm{psi})$ for $2 \mathrm{~h}$. Reaction mixture was filtered through celite bed and concentrated the filtrate under reduced pressure to obtain 2-(8-Amino-octyl)-6methoxy-benzoic acid methyl ester (6) as a yellow liquid; Yield: $1.7 \mathrm{~g}$ (92.5\%); IR (neat): $v_{\max } 3436,2931$,
2857, 1729, 1587, 1467, 1438, 1268, 1111, 1073, 829, $753 \mathrm{~cm}^{-1} ;{ }^{1} \mathrm{H}$ NMR $\left(400 \mathrm{MHz}, \mathrm{CDCl}_{3}: \delta 1.273\right.$ (brs, $8 \mathrm{H}), 1.54$ (brs, $2 \mathrm{H}), 1.65-1.79(\mathrm{~m}, 2 \mathrm{H}), 2.51$ (t, $2 \mathrm{H}, J=$ $7.6 \mathrm{~Hz}), 2.94(\mathrm{t}, 2 \mathrm{H}, J=8.0 \mathrm{~Hz}), 3.79(\mathrm{~s}, 3 \mathrm{H}), 3.89$ (s, $3 \mathrm{H}), 6.74(\mathrm{~d}, 1 \mathrm{H}, J=8.0 \mathrm{~Hz}), 6.80(\mathrm{~d}, 1 \mathrm{H}, J=8.0 \mathrm{~Hz})$, 7.23-7.27 (m, 1H) ppm; ESIMS(m/z): $294(\mathrm{M}+\mathrm{H})^{+}$.

\subsection{Synthesis of sulphonamides (7)}

A solution of amine $6(300 \mathrm{mg}, 1.023 \mathrm{mmol})$ in dry dichloromethane (DCM) was added to triethyl amine (TEA) $(2.55 \mathrm{mmol})$ followed by sulphonyl chlorides $(1.22 \mathrm{mmol})$ at $0^{\circ} \mathrm{C}$. The contents were slowly brought to room temperature and stirred for $2 \mathrm{~h}$. Reaction mixture was diluted with DCM and washed with water and brine solution dried and distilled off and obtained crude compound was purified by column.

2.2a Synthesis of methyl 2-methoxy-6-(8(methylsulfonamideo)octyl)benzoate (7a): Using 6 and methane sulphonyl chloride as starting materials, the title compound 7a was obtained as a light yellow liquid; Yield: $96.5 \%$; IR (neat): $v_{\max } 3294,3087,3009$, 2932, 2856, 1728, 1667, 1588, 1464, 1434, 1320, 1268, $1151,1107,1072,970,826 \mathrm{~cm}^{-1} ;{ }^{1} \mathrm{H}$ NMR $(400 \mathrm{MHz}$, $\left.\mathrm{CDCl}_{3}\right): \delta 1.29$ (brs, 8H), 1.53-1.59 (m, 4H), 2.53 $(\mathrm{t}, 2 \mathrm{H}, \mathrm{J}=7.8 \mathrm{~Hz}), 2.95(\mathrm{~s}, 3 \mathrm{H}), 3.09-3.14(\mathrm{~m}, 2 \mathrm{H})$, $3.82(\mathrm{~s}, 3 \mathrm{H}), 3.91(\mathrm{~s}, 3 \mathrm{H}), 4.23(\mathrm{brs}, 1 \mathrm{H}), 6.76(\mathrm{~d}, 1 \mathrm{H}, J$ $=8.8 \mathrm{~Hz}), 6.81(\mathrm{~d}, 1 \mathrm{H}, J=7.6 \mathrm{~Hz}), 7.25-7.29(\mathrm{~m}, 1 \mathrm{H})$ ppm; ${ }^{13} \mathrm{CNMR}\left(100 \mathrm{MHz}, \mathrm{CDCl}_{3}\right): \delta 26.37,28.84$, 29.06, 29.14, 29.94, 30.90, 33.29, 39.99, 43.19, 52.08, 55.77, 108.33, 121.38, 123.34, 130.19, 141.09, 156.14, 168.89 ppm; MF: $\mathrm{C}_{18} \mathrm{H}_{29} \mathrm{NO}_{5} \mathrm{~S}$; Exact Mass: 371.18; $\operatorname{ESIMS}(\mathrm{m} / \mathrm{z}): 372.1(\mathrm{M}+\mathrm{H})^{+}$.

2.2b Synthesis of methyl 2-methoxy-6-(8(phenylsulfonamido)octyl)benzoate (7b): Using 6 and benzene sulphonyl chloride as starting materials, the title compound $\mathbf{7 b}$ was obtained as a light pale yellow liquid; Yield: $90.2 \%$; IR (neat): $\nu_{\max } 3288,3064,3003$, 2932, 2856, 1726, 1588, 1459, 1322, 1268, 1162, 1099, $1078,859 \mathrm{~cm}^{-1} ;{ }^{1} \mathrm{H}$ NMR $\left(400 \mathrm{MHz}, \mathrm{CDCl}_{3}\right): \delta 1.21$ (brs, 8H), 1.41-1.45 (m, 2H), 1.51-1.56 (m, 2H) 2.52 $(\mathrm{t}, 2 \mathrm{H}, J=7.2 \mathrm{~Hz}), 2.92-2.95(\mathrm{~m}, 2 \mathrm{H}), 3.81(\mathrm{~s}, 3 \mathrm{H})$, $3.82(\mathrm{~s}, 3 \mathrm{H}), 4.38(\mathrm{~s}, 1 \mathrm{H}), 6.76(\mathrm{~d}, 1 \mathrm{H}, J=8.4 \mathrm{~Hz})$, $6.80(\mathrm{~d}, 1 \mathrm{H}, J=7.6 \mathrm{~Hz}), 7.25-7.29(\mathrm{~m}, 1 \mathrm{H}), 7.49-7.59$ $(\mathrm{m}, 3 \mathrm{H}), 7.86(\mathrm{~d}, 2 \mathrm{H}, J=7.6 \mathrm{~Hz}) \mathrm{ppm} ;{ }^{13} \mathrm{C} \mathrm{NMR}$ $\left(100 \mathrm{MHz}, \mathrm{CDCl}_{3}\right): \delta 26.7,29.1,29.2,29.3,29.6,31.2$, $36.2,42.8,51.5,55.8,111.4,116.0,120.3,129.0$, $130.3,131.9,136.8,144.5,156.3,168.2 \mathrm{ppm}$; MF: $\mathrm{C}_{23} \mathrm{H}_{31} \mathrm{NO}_{5} \mathrm{~S}$; Exact Mass: 433.19; ESIMS (m/z): 432.0 $(\mathrm{M}-\mathrm{H})^{+}$. 
2.2c Synthesis of methyl 2-methoxy-6-(8(2methylphenyl sufonamido) octyl) benzoate (7c): Using $\mathbf{6}$ and 2-methylphenylsulphonyl chloride as starting materials, the title compound $\mathbf{7 c}$ was obtained as a yellow liquid; Yield: 74.4\%; IR (neat): $v_{\max } 3304$, 3061, 3007, 2932, 2855, 1728, 1588, 1464, 1431, 1318, 1270, 1158, 1112, 1073, 959, $755 \mathrm{~cm}^{-1} ;{ }^{1} \mathrm{H}$ NMR $\left(400 \mathrm{MHz}, \mathrm{CDCl}_{3}\right): \delta 1.18$ (brs, $\left.8 \mathrm{H}\right), 1.39-1.57$ (m, $4 \mathrm{H}), 2.51(\mathrm{t}, 2 \mathrm{H}, J=7.6 \mathrm{~Hz}), 2.64(\mathrm{~s}, 3 \mathrm{H}), 2.89-2.94$ $(\mathrm{m}, 2 \mathrm{H}), 3.82(\mathrm{~s}, 3 \mathrm{H}), 3.90(\mathrm{~s}, 3 \mathrm{H}), 4.42(\mathrm{~s}, 1 \mathrm{H}), 6.75-$ $6.81(\mathrm{~m}, 2 \mathrm{H}), 7.25-7.32(\mathrm{~m}, 3 \mathrm{H}), 7.42-7.46(\mathrm{~m}, 1 \mathrm{H})$, 7.95-7.97 (m, 1H) ppm; ${ }^{13} \mathrm{C}$ NMR $\left(100 \mathrm{MHz}, \mathrm{CDCl}_{3}\right)$ : $22.0,26.7,29.1,29.2,29.3,29.6,31.3,36.2,42.8$, $51.5,55.8,111.4,116.0,120.3,120,8,129.7,130.5$, 131.8, 136.6, 136.8, 138.9, 146.9, 156.3, $168.2 \mathrm{ppm}$; MF: $\mathrm{C}_{24} \mathrm{H}_{33} \mathrm{NO}_{5} \mathrm{~S}$; Exact Mass: 447.21; ESIMS (m/z): $448.1(\mathrm{M}+\mathrm{H})^{+}$.

2.2d Synthesis of methyl 2-methoxy-6-(8(methylphenylsulfonamido)octyl) benzoate (7d): Using 6 and para toluene sulphonyl chloride as starting materials, the title compound 7d was obtained as a light yellow liquid; Yield: 98\%; IR (neat): $v_{\max } 3285,3050$, 2931, 2856, 1728, 1589, 1464, 1436, 1322, 1269, 1157, 1104, 1076, $817 \mathrm{~cm}^{-1} ;{ }^{1} \mathrm{H}$ NMR (400 MHz, $\left.\mathrm{CDCl}_{3}\right): \delta$ 1.213 (brs, 8H), 1.39-1.60 (m, 4H), 2.42 (s, 3H), 2.51 (t, $2 \mathrm{H}, J=7.6 \mathrm{~Hz}), 2.88-2.96(\mathrm{~m}, 2 \mathrm{H}) 3.81(\mathrm{~s}, 3 \mathrm{H})$, $3.90(\mathrm{~s}, 3 \mathrm{H}), 4.28(\mathrm{~s}, 1 \mathrm{H}), 6.76(\mathrm{~d}, 1 \mathrm{H}, J=8.4 \mathrm{~Hz})$, $6.80(\mathrm{~d}, 1 \mathrm{H}, J=7.6 \mathrm{~Hz}), 7.25-7.31(\mathrm{~m}, 3 \mathrm{H}), 7.73(\mathrm{~d}$, $2 \mathrm{H}, J=8.4 \mathrm{~Hz}) \mathrm{ppm} ;{ }^{13} \mathrm{C} \mathrm{NMR}\left(100 \mathrm{MHz}, \mathrm{CDCl}_{3}\right)$ : $\delta$ 21.43, 26.37, 28.80, 29.06, 29.17, 29.45, 30.94, $33.34,43.12,52.10,55.82,108.35,121.41,123.4$, 127.04, 129.6, 130.21, 136.99, 141.15, 143.21, 156.2, 168.9 ppm; MF: $\mathrm{C}_{24} \mathrm{H}_{33} \mathrm{NO}_{5} \mathrm{~S}$; Exact Mass: 447.21; $\operatorname{ESIMS~(m/z):~} 446.1(\mathrm{M}-\mathrm{H})^{+}$.

2.2e Synthesis of methyl 2-(8-(2,5dimethylphenylsufonamido)octyl)-6-methoxybenzoate (7e): Using 6 and 2,5dimethylphenylsulphonyl chloride as starting materials, the title compound $7 \mathbf{e}$ was obtained as a yellow liquid; Yield: $74.1 \%$; IR (neat): $v_{\max } 3305,3066,3013,2932,2856,1728,1589,1464$, 1429, 1317, 1268, 1151, 1105, 1073, 967, $896 \mathrm{~cm}^{-1}$; ${ }^{1} \mathrm{H}$ NMR $\left(400 \mathrm{MHz}, \mathrm{CDCl}_{3}\right): \delta 1.19$ (brs, 8H), 1.39$1.44(\mathrm{~m}, 2 \mathrm{H}), 1.50-1.57(\mathrm{~m}, 2 \mathrm{H}), 2.36(\mathrm{~s}, 3 \mathrm{H}), 2.52(\mathrm{t}$, $2 \mathrm{H}, J=7.6 \mathrm{~Hz}), 2.58(\mathrm{~s}, 3 \mathrm{H}), 2.87-2.93(\mathrm{q}, 2 \mathrm{H}), 3.82$ (s, 3H), 3.90 (s, 3H), $4.40(\mathrm{bs}, 1 \mathrm{H}), 6.75-6.81(\mathrm{~m}, 2 \mathrm{H})$, 7.17-7.19 (m, 1H), 7.23-7.288 (m, 2H), $7.78(\mathrm{~s}, 1 \mathrm{H})$ ppm; MF: $\mathrm{C}_{25} \mathrm{H}_{35} \mathrm{NO}_{5} \mathrm{~S}$; Exact Mass: $461.22 ;{ }^{13} \mathrm{C}$ NMR $\left(100 \mathrm{MHz}, \mathrm{CDCl}_{3}\right): 21.3,22.0,26.7,29.1,29.2,29.3$, 29.6, 31.2, 36.2, 42.8, 51.5, 55.8, 111.4, 116.0, 120.3,
126.6, 129.2, 132.1, 133.6, 135.7, 136.8, 138.8, 146.9, 156.3, 168.2 ppm; ESIMS (m/z): $462.1(\mathrm{M}+\mathrm{H})^{+}$.

$2.2 \mathrm{f}$ Synthesis of methyl 2-(8-(2,5dichlorophenylsulfonamido)octyl)-6-methoxybenzoate (7f): Using 6 and 2,5-dichloro phenyl sulphonyl chloride as starting materials, the title compound $7 \mathbf{f}$ was obtained as a pale yellow liquid; Yield: $60.4 \%$; IR (neat): $v_{\max } 3310,3085,3008,2932,2856,1726,1586$, 1456, 1334, 1268, 1164, 1101, 1078, 959, $894 \mathrm{~cm}^{-1} ;{ }^{1} \mathrm{H}$ NMR (400 MHz, $\left.\mathrm{CDCl}_{3}\right): \delta 1.22$ (brs, $\left.8 \mathrm{H}\right), 1.43-1.56$ $(\mathrm{m}, 4 \mathrm{H}), 2.52(\mathrm{t}, 2 \mathrm{H}, J=7.2 \mathrm{~Hz}), 2.91-2.96(\mathrm{~m}, 2 \mathrm{H})$, 3.82 (s, 3H), 3.91 (s, 3H), 4.96 (brs, 1H), 6.75-6.82 (m, 2H), 7.26-7.29 (m, 1H), 7.46 (brs, 2H), 8.09 (s, 1H) ppm; ${ }^{13} \mathrm{C}$ NMR $\left(100 \mathrm{MHz}, \mathrm{CDCl}_{3}\right): \delta 26.30,28.75$, 29.06, 29.16, 29.25, 29.30, 29.39, 30.93, 33.34, 43.32, 52.12, 55.82, 108.37, 121.41, 123.41, 129.47, 130.23, $131.09,132.59,133.42,133.46,138.72,141.13$, 156.22, 168.91 ppm; MF: $\mathrm{C}_{23} \mathrm{H}_{29} \mathrm{Cl}_{2} \mathrm{NO}_{5} \mathrm{~S}$; Exact Mass: 501.11; ESIMS (m/z): $500.0(\mathrm{M}-\mathrm{H})^{+} .502 .0$ (chloro).

$2.2 \mathrm{~g}$ Synthesis of methyl 2-methoxy-6-(8(phenylmethylsulfonamido) octyl) benzoate (7g): Using 6 and phenylmethylsulphonyl chloride as starting materials, the title compound $\mathbf{7 g}$ was obtained as a pale yellow liquid; Yield: $26.8 \%$; IR (neat): $v_{\max }$ 3294, 3072, 3034, 2931, 2856, 1727, 1667, 1587, 1461, 1320, 1268, 1147, 1117, 1074, $957 \mathrm{~cm}^{-1} ;{ }^{1} \mathrm{H}$ NMR $\left(400 \mathrm{MHz}, \mathrm{CDCl}_{3}\right): \delta 1.24$ (brs, $\left.8 \mathrm{H}\right) 1.34-1.56(\mathrm{~m}$, $4 \mathrm{H}), 2.53(\mathrm{t}, 2 \mathrm{H}, J=7.6 \mathrm{~Hz}), 2.94-2.99(\mathrm{~m}, 2 \mathrm{H}), 3.82$ (s, 3H), $3.90(\mathrm{~s}, 3 \mathrm{H}), 4.00(\mathrm{~s}, 3 \mathrm{H}), 4.25(\mathrm{~s}, 2 \mathrm{H}), 6.76$ $(\mathrm{d}, 1 \mathrm{H}, J=8.4 \mathrm{~Hz}), 6.81(\mathrm{~d}, 1 \mathrm{H}, J=7.6 \mathrm{~Hz}), 7.3(\mathrm{~m}$, 1H), 7.38 (brs, 5H) ppm; ${ }^{13} \mathrm{C} \mathrm{NMR}\left(100 \mathrm{MHz}, \mathrm{CDCl}_{3}\right)$ : 26.7, 29.1, 29.2, 29.3, 29.6, 31.2, 36.2, 43.2, 51.5, 55.8, 66.0, 111.4, 116.0, 120.3, 125.7, 128.6, 130.8, 133.2, 136.8, 146.9, 156.3, 168.2 ppm; MF: $\mathrm{C}_{24} \mathrm{H}_{33} \mathrm{NO}_{5} \mathrm{~S}$; Exact Mass: 447.21; ESIMS (m/z): $446.1(\mathrm{M}-\mathrm{H})^{+}$.

2.2h Synthesis of methyl 2-methoxy-6-(8(naphthalene-2-sulfonamido) octyl) benzoate (7h): Using 6 and naphthalene-2-sulphonyl chloride as starting materials, the title compound $\mathbf{7 h}$ was obtained as a light yellow liquid; Yield: $82.9 \%$; IR (neat): 3286, 3056, 2929, 2855, 1726, 1587, 1511, 1461, 1323, 1268, 1156, 1111, 1074, 960, $903 \mathrm{~cm}^{-1}$; ${ }^{1} \mathrm{H}$ NMR $(400 \mathrm{MHz}$, $\mathrm{CDCl}_{3}$ ): $\delta 1.187$ (broad singlet, $\left.8 \mathrm{H}\right), 1.42-1.52(\mathrm{~m}$, $4 \mathrm{H}), 2.49(\mathrm{t}, 2 \mathrm{H}, J=7.6 \mathrm{~Hz}), 2.95-2.99(\mathrm{~m}, 2 \mathrm{H}), 3.82$ (s, 3H), 3.89 (s, 3H), 4.45 (brs, 1H), 6.75-6.80 (m, 2H), 7.24-7.28 (m, 1H), 7.59-7.66 (m, 2H), 7.82-8.02 (m, $4 \mathrm{H}), 8.44$ (s, $1 \mathrm{H})$ ppm; MF: $\mathrm{C}_{27} \mathrm{H}_{33} \mathrm{NO}_{5} \mathrm{~S}$; Exact Mass: 483.21; ${ }^{13} \mathrm{C}$ NMR $\left(100 \mathrm{MHz}, \mathrm{CDCl}_{3}\right): 26.7,29.1,29.2$, 
29.3, 29.6, 31.2, 36.2, 42.8, 51.8, 55.8, 111.4, 116.0, $120.3,123.4,126.0,126.2,128.1,129.4,134.1,136.7$, 137.0, 146.9, 156.3, 168.2 ppm; ESIMS (m/z): 482.0 $(\mathrm{M}-\mathrm{H})^{+}$.

2.2i Synthesis of methyl 2-methoxy-6-(8-(thiophene2-sulfonamido) octyl) benzoate (7i): Using 6 and thiophene-2- sulphonyl chloride as starting materials, the title compound $7 \mathbf{i}$ was obtained as a light yellow liquid; Yield: $68.9 \%$; IR (neat): $v_{\max } 3285,3099,2931$, 2856, 1726, 1586, 1463, 1426, 1331, 1269, 1157, 1108, 1073, 1018, $728 \mathrm{~cm}^{-1} ;{ }^{1} \mathrm{H}$ NMR $\left(400 \mathrm{MHz}, \mathrm{CDCl}_{3}\right): \delta$ 1.23 (brs, 8H), 1.44-1.56 (m, 4H), $2.52(\mathrm{t}, 2 \mathrm{H}, J=$ 7.6 Hz), 3.00-3.05 (m, 2H), $3.82(\mathrm{~s}, 3 \mathrm{H}), 3.91$ (s, 3H), 4.46 (brs, $1 \mathrm{H}), 6.76(\mathrm{~d}, 1 \mathrm{H}, J=8.4 \mathrm{~Hz}), 6.81(\mathrm{~d}$, $1 \mathrm{H}, J=8 \mathrm{~Hz}), 7.07-7.09(\mathrm{~m}, 1 \mathrm{H}), 7.25-7.29(\mathrm{~m}$, $1 \mathrm{H}), 7.57-7.60(\mathrm{~m}, 2 \mathrm{H}) \mathrm{ppm} ;{ }^{13} \mathrm{C} \mathrm{NMR}(100 \mathrm{MHz}$, $\left.\mathrm{CDCl}_{3}\right): 26.7,29.1,29.2,29.3,29.6,31.2,36.2,42.9$, 51.8, 55.8, 111.4, 116.0, 120.3, 125.8, 126.0, 127.2, 136.8, 146.9, 156.3, 168.2 ppm; MF: $\mathrm{C}_{21} \mathrm{H}_{29} \mathrm{NO}_{5} \mathrm{~S}_{2}$; Exact Mass: 439.15; ESIMS (m/z):438.0 (M-H) ${ }^{+}$.

$2.2 \mathrm{j}$ Synthesis of methyl 2-(8-(5-bromo-thiophene-2sulfonamido)octyl)-6-methoxy-benzoate (7j): Using 6 and 5-bromo thiophene-2- sulphonyl chloride as starting materials, the title compound $\mathbf{7} \mathbf{j}$ was obtained as a light yellow liquid; Yield: 69.9\%; IR (neat): $v_{\max }$ 3282, 3097, 2931, 2856, 1726, 1664, 1586, 1463, 1411, 1333, 1268, 1156, 1075, 1023, 966, $805 \mathrm{~cm}^{-1} ;{ }^{1} \mathrm{H}$ NMR $\left(400 \mathrm{MHz}, \mathrm{CDCl}_{3}\right): \delta 1.25$ (brs, $\left.8 \mathrm{H}\right), 1.45-1.56(\mathrm{~m}, 4 \mathrm{H})$, $2.53(\mathrm{t}, 2 \mathrm{H}, J=7.6 \mathrm{~Hz}), 2.99-3.05(\mathrm{~m}, 1 \mathrm{H}), 3,82(\mathrm{~s}$, $3 \mathrm{H}), 3.91(\mathrm{~s}, 3 \mathrm{H}), 4.52(\mathrm{~s}, 1 \mathrm{H}), 6.76(\mathrm{~d}, 1 \mathrm{H}, J=$ $8.4 \mathrm{~Hz}), 6.81(\mathrm{~d}, 1 \mathrm{H}, J=7.6 \mathrm{~Hz}), 7.06(\mathrm{~d}, 1 \mathrm{H}, J=$ $4 \mathrm{~Hz}), 7.26-7.29(\mathrm{~m}, 1 \mathrm{H}), 7.35(\mathrm{~d}, 1 \mathrm{H}, J=4 \mathrm{~Hz})$ ppm; ${ }^{13} \mathrm{C}$ NMR $\left(100 \mathrm{MHz}, \mathrm{CDCl}_{3}\right): 26.7,29.1,29.2$, 29.3, 29.6, 31.2, 36.2, 42.9, 51.8, 55.8, 111.4, 111.9, 116.0, 120.3, 128.0, 128.7, 130.3, 136.8, 146.9, 156.3, 168.2 ppm; MF: $\mathrm{C}_{21} \mathrm{H}_{28} \mathrm{BrNO}_{5} \mathrm{~S}_{2}$; Exact Mass: 517.06; $\operatorname{ESIMS~(m/z):~} 517.9(\mathrm{M}+\mathrm{H})^{+} .519 .9$ (bromo).

2.2k Synthesis of methyl 2-(8-(1-(5-fluoropyridin-2-yl)1 H-pyrazole-5-sulfonamido)octyl)-6-methoxybenzoate (7k): Using 6 and 1-(5-fluoropyridin-2-yl)-1Hpyrazole-5-sulfonyl chloride as starting materials, the title compound $\mathbf{7 k}$ was obtained as a off-white solid; Yield: 93.7\%; MP: $76-77^{\circ} \mathrm{C}$; IR (neat): $v_{\max } 3234$, $3119,3069,3002,2941,2856,1725,1583,1511$, $1445,1325,1275,1180,1152,1131,1085 \mathrm{~cm}^{-1} ;{ }^{1} \mathrm{H}$ NMR $\left(400 \mathrm{MHz}, \mathrm{CDCl}_{3}\right): \delta 1.24$ (brs, $\left.8 \mathrm{H}\right), 1.47-1.52$ $(\mathrm{m}, 4 \mathrm{H}), 2.51(\mathrm{t}, 2 \mathrm{H}, J=8.0 \mathrm{~Hz}), 3.00-3.05(\mathrm{q}, 2 \mathrm{H})$, $3.81(\mathrm{~s}, 3 \mathrm{H}), 3.90(\mathrm{~s}, 3 \mathrm{H}), 4.49(\mathrm{~s}, 1 \mathrm{H}), 6.74-6.82$ (m, 2H), 7.24-7.28 (m, 1H), 7.56-7.61 (m, 1H), 7.95 (s, 1H), 7.99-8.02 (m, 1H), $8.288(\mathrm{~s}, 1 \mathrm{H}), 8.88(\mathrm{~s}, 1 \mathrm{H})$ ppm; ${ }^{13} \mathrm{C} \mathrm{NMR}\left(100 \mathrm{MHz}, \mathrm{CDCl}_{3}\right): 26.7,29.1,29.2$, 29.3, 29.6, 31.2, 36.2, 42.9, 51.8, 55.8, 110.9, 111.4, 113.3, 116.0, 120.3, 121.1, 134.5, 136.8, 139.8, 146.9, 147.6, 154.5, 156.3, 168.2 ppm; MF: $\mathrm{C}_{25} \mathrm{H}_{31} \mathrm{FN}_{4} \mathrm{O}_{5} \mathrm{~S}$; Exact Mass: 518.20; $\quad$ ESIMS $\quad(\mathrm{m} / \mathrm{z}): \quad 517.1$ $(\mathrm{M}-\mathrm{H})^{+}$.

2.21 Synthesis of methyl 2-(8-(1-(4-fluorophenyl)-1Hpyrazole-5-sulfonamido) octyl) -6-methoxybenzoate (7l): Using 6 and 1-(4-fluorophenyl)-1H-pyrazole-5sulfonyl chloride as starting materials, the title compound 7l was obtained as a pale brown liquid; Yield: 70.8\%; IR (KBr pellet): $v_{\max } 3284,3128,3080,3004$, 2932, 2856, 1726, 1587, 1521, 1435, 1323, 1270, 1171, 1146, 1102, 1073, 954, $837 \mathrm{~cm}^{-1}$; ${ }^{1} \mathrm{H}$ NMR $(400 \mathrm{MHz}$, $\mathrm{CDCl}_{3}$ ): $\delta 1.26$ (brs, $\left.8 \mathrm{H}\right), 1.45-1.56(\mathrm{~m}, 4 \mathrm{H}), 2.52(\mathrm{t}$, $2 \mathrm{H}, J=7.2 \mathrm{~Hz}), 3.01-3.05(\mathrm{q}, 2 \mathrm{H}), 3.82(\mathrm{~s}, 3 \mathrm{H}), 3.90$ (s, 3H), $4.5(\mathrm{~s}, 1 \mathrm{H}), 6.75-6.81(\mathrm{~m}, 2 \mathrm{H}), 7.16-7.26(\mathrm{~m}$, $3 \mathrm{H}), 7.63-7.67(\mathrm{~m}, 2 \mathrm{H}), 7.95(\mathrm{~s}, 1 \mathrm{H}), 8.26(\mathrm{~s}, 1 \mathrm{H}) ;{ }^{13} \mathrm{C}$ NMR $\left(100 \mathrm{MHz}, \mathrm{CDCl}_{3}\right): 26.7,29.1,29.2,29.3,29.6$, 31.2, 36.2, 42.9, 51.8, 55.8, 111.4, 113.5, 115.6, 116.1, 120.3, 132.7, 135.3, 136.8, 141.9, 146.9, 156.3, 160.4, 168.2 ppm; MF: $\mathrm{C}_{26} \mathrm{H}_{32} \mathrm{FN}_{3} \mathrm{O}_{5} \mathrm{~S}$; Exact Mass: 517.20; $\operatorname{ESIMS~(m/z)~ppm:~} 518.3(\mathrm{M}+\mathrm{H})^{+}$.

\subsection{Antibacterial bioassay}

Sulfonamide derivatives of anacardic acid (7a-7l) were dissolved in dimethyl sulphoxide at $250 \mu \mathrm{g} / \mathrm{mL}$ concentration. The composition of nutrient agar medium was bactotryptone $(10 \mathrm{~g})$, yeast extract $(5 \mathrm{~g}), \mathrm{NaCl}(10 \mathrm{~g})$, final $\mathrm{pH} 7.4$. After $18 \mathrm{~h}$ the exponentially growing cultures of the six bacteria in nutrient broth at $37^{\circ} \mathrm{C}$ were diluted in sterile broth. From each of these diluted cultures, $1 \mathrm{~mL}$ was added to $100 \mathrm{~mL}$ sterilized and cooled nutrient agar media to give a final bacterial count of $1 \times 10^{6} \mathrm{cell} / \mathrm{ml}$. The plates were set at room temperature and later dried at $37^{\circ} \mathrm{C}$ for $20 \mathrm{~h}$. Paper discs $(6 \mathrm{~mm}$, punched from Whatmann No. 41 paper) were ultraviolet sterilized and used for the assays. Discs were soaked in different concentration of the test solution and placed on the inoculated agar media at regular intervals of 6-7 cm, care was taken to ensure that excess solution was not on the discs. All the samples were taken in triplicates. The plates were incubated at $37^{\circ} \mathrm{C}$ in an inverted fashion. Activity was determined by zones showing complete inhibition (mm). Growth inhibition was calculated with reference to positive control. 


\section{Results and discussion}

We described here the synthesis of various biologically active novel sulfonamide derivatives at C-8 alkyl chain using anacardic acid mixture as starting material and various reagents in the given below conditions (scheme 1).

As shown in scheme 1, the heterogeneous mixture of anacardic acids (1)(1a-d) was isolated from commercially available CNSL by a reported method. ${ }^{26}$ Accordingly, CNSL was treated with calcium hydroxide, during which anacardic acid present in CNSL becomes calcium anacardate, which was isolated and hydrolysed with diluted hydrochloric acid to generate anacardic acid ene mixture, which was a mixture of monoene, diene and triene located at $\left(8^{\prime}\right),\left(8^{\prime}, 11^{\prime}\right)$ and $\left(8^{\prime}, 11\right.$ ', 14 ') of the $\mathrm{C} 15$ alkyl chain, respectively. The mixture of anacardic acids (1) was quantitatively converted into $\mathbf{2}$, on a multigram scale, by treatment with dimethyl sulphate under phase transfer catalysis. The mixture of dialkylated anacardic acids was submitted to ozonolysis, followed by reductive cleavage of the derived ozonides with sodium borohydride, furnishing the alcohol 3. The resultant alcohol compound $\mathbf{3}$ was further converted to bromo compound $\mathbf{4}$ by treatment with $\mathrm{CBr}_{4}$ in the presence of pyridine and TPP in $\mathrm{CH}_{2} \mathrm{Cl}_{2}$ at $0^{\circ} \mathrm{C}$ at room temperature for $8 \mathrm{~h}$. Resultant bromo compound 4 was reacted with sodium azide followed by reduction with $\mathrm{Pd} / \mathrm{C}$ under $\mathrm{H}_{2}$ pressure to obtain amine compound $\mathbf{6}$, which was coupled with various sulfonyl chlorides in the presence of triethylamine in DCM to obtain compounds (7a-7l, scheme 1) of sulfonamide derivatives at C-8 alkyl chain were purified by column chromatography to yield title compounds. The structure of sulfonamide derivatives (7a-7l) was determined by using different spectroscopic techniques IR, ${ }^{1} \mathrm{H}$ NMR and Mass. The resulting compounds were screened for their antibacterial activity.

\subsection{Biological activity}

The sulfonamide derivatives (7a-7l) were screened for their antibacterial activity ${ }^{27}$ against some of the pathogenic bacteria viz. E. coli (MTCC443), P. aeruginosa (MTCC424), S. aureus (MTCC96) and S. pyogenes (MTCC443) using agar well diffusion method according to the literature protocol. ${ }^{26}$ The antibacterial activity of the analogues was compared with standard drug ampicilline and the results of investigation have been presented in table 1 . It is observed that the most of the compounds showed high biological activity. Based on the test results, it is evident that several of synthesized anacardic acid analogues possess moderate to good activity against the gram +ve and gram -ve bacteria. Of all the compounds prepared, entities

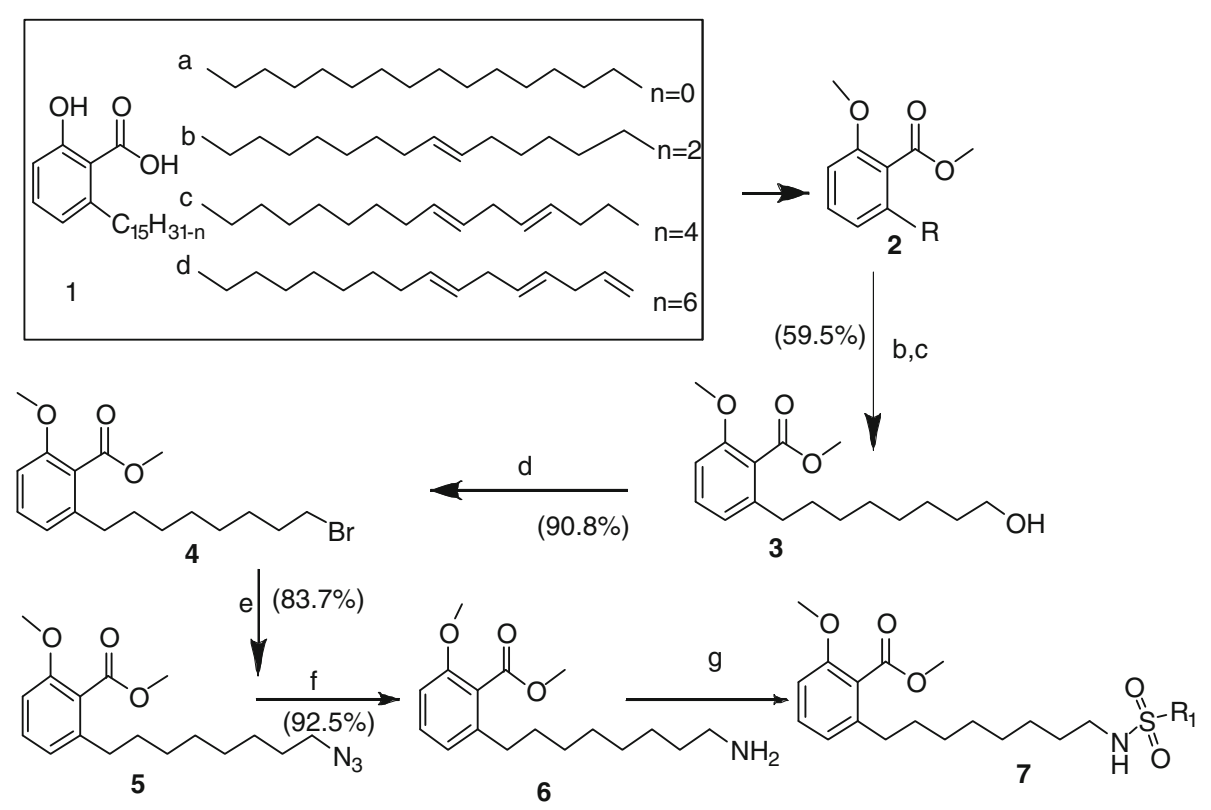

Scheme 1. Synthesis of various biologically active sulfonamides from anacardic acid mixture. Reagents: (a) di methyl sulfate, $\mathrm{K}_{2} \mathrm{CO}_{3}, \mathrm{ACN}, 90^{\circ} \mathrm{C}, 24 \mathrm{~h}$; (b) ozonolysis, $\mathrm{MeOH}, \mathrm{CH}_{2} \mathrm{Cl}_{2},-78^{\circ} \mathrm{C}, 6 \mathrm{~h}$; (c) $\mathrm{MeOH}, \mathrm{NaBH}_{4}, 18 \mathrm{~h}, 0^{\circ} \mathrm{C}$, R.T; (d) $\mathrm{CBr}_{4}$, Pyridine, TPP, $\mathrm{CH}_{2} \mathrm{Cl}_{2}, 0^{\circ} \mathrm{C}$, R.T, 8 h; (e) $\mathrm{NaN}_{3}$, DMF, $100^{\circ} \mathrm{C}, 4 \mathrm{~h}$; (f) $10 \% \mathrm{Pd} / \mathrm{C}, 50 \mathrm{psi}$, $2 \mathrm{~h}$; (g) different sulphonyl chlorides, TEA, DCM. 
Table 1. Antibacterial activity of sulfonamide derivatives of anacardic acid (7a-7l).

\begin{tabular}{|c|c|c|c|c|c|}
\hline \multirow[b]{2}{*}{ S. No. } & \multirow[b]{2}{*}{$\mathrm{R}_{1}$} & \multicolumn{4}{|c|}{$\begin{array}{c}\text { Name of the bacteria (Conc. } 250 \mu \mathrm{g} / \mathrm{ml} \text { ) and } \\
\text { inhibition zone in } \mathrm{mm}\end{array}$} \\
\hline & & $\begin{array}{c}\text { E. coli } \\
\text { MTCC443 }\end{array}$ & $\begin{array}{l}\text { P. aeruginosa } \\
\text { MTCC424 }\end{array}$ & $\begin{array}{l}\text { S. aureus } \\
\text { MTCC96 }\end{array}$ & $\begin{array}{l}\text { S. pygenes } \\
\text { MTCC } 442\end{array}$ \\
\hline $7 \mathbf{a}$ & & 19 & 18 & 16 & 16 \\
\hline $7 b$ & & 18 & 17 & 15 & 17 \\
\hline $7 \mathrm{c}$ & & 20 & 18 & 17 & 17 \\
\hline 7d & & 22 & 20 & 17 & 18 \\
\hline 7e & & 17 & 22 & 17 & 19 \\
\hline $7 f$ & CI & 21 & 19 & 17 & 17 \\
\hline $7 \mathrm{~g}$ & & 20 & 19 & 16 & 18 \\
\hline $7 \mathrm{~h}$ & & 19 & 18 & 18 & 18 \\
\hline $7 \mathbf{i}$ & & 22 & 19 & 16 & 16 \\
\hline $7 \mathbf{j}$ & & 23 & 21 & 17 & 18 \\
\hline $7 \mathbf{k}$ & & 21 & 19 & 17 & 17 \\
\hline 71 & $\vec{F}$ & 19 & 20 & 17 & 17 \\
\hline $\begin{array}{l}\mathrm{SD}^{*} \\
\text { amplicilline }\end{array}$ & SD* amplicilline & 20 & 20 & 18 & 19 \\
\hline
\end{tabular}

Standard indicated by star

7d, 7f, 7g, 7i, 7j and 7k, of E. coli MTCC443, 7d, 7e, $7 \mathbf{j}$ and $7 \mathbf{l}$ of $P$. aeruginosa MTCC424, 7h, 7k and $7 \mathbf{l}$ of $S$. aureus MTCC96 and $\mathbf{7 e}, \mathbf{7 g}$, and $\mathbf{7 h}$ of $S$. pyogenes MTCC442 displayed good to excellent activity while the remaining compounds showed moderate activity. The most active antibacterial agent against Escherichia coli found to be compound $7 \mathbf{i}$ and $\mathbf{7} \mathbf{j}$ having thiophene compound and other compounds in the series exhibited moderate to good activity. The activity depends on the $\mathrm{R}$ substituent to some extent, however all the compounds show antibacterial activity. So, other functionalities in the molecule will contribute to the activity as well. It 
may be suggested that the anacardic acid derivative with a suitable $\mathrm{R}$ may lead to a good antibacterial agent against all the Escherichia coli, Pseudomonas aeruginosa, Staphylococcus aureus and Streptococcus pyogenes bacterial strains.

\section{Conclusion}

In summary, the present study describes a convenient and efficient protocol for the synthesis of sulfonamide derivatives by using anacardic acid mixture using various reagents and different conditions. We believe that this procedure is convenient, economic and a userfriendly process for the synthesis of these various sulphonamide compounds from anacardic acid mixture. All compounds structures were supported by IR, NMR, Mass spectral data. Sulphonamide derivatives were screened for their antibacterial activity against few bacterial strains and it was observed that some of the compounds showed more biological activity.

\section{Acknowledgements}

We thank GVK Biosciences Private Limited for the financial support and encouragement. We also thank the Analytical Department for their analytical data and to Dr. Balaram Patro for his helpful suggestions.

\section{References}

1. Tyman J H P 1979 Chem. Soc. Rev. 8499

2. Kubo I, Muroi H, Himejima M and Yamigiwa Y 1993 J. Agric. Food Chem. 411016

3. Gillerman J L, Walsh N J, Werner N K and Schlenk H 1969 Can. J. Microbiol. 151219

4. Shoba S V, Ramadoss C S and Ravindranath B 1994 J. Nat. Prod. 571755

5. Ha T J and Kubo I 2005 J. Agric. Food Chem. 534350

6. Chandregowda V, Kush A and Reddy G 2009 Eur. J. Med. Chem. 442711

7. Paramashivappa R, Phanikumar P, Subba Rao P V and Srinivasa Rao A 2002 J. Agric. Food Chem. 507709

8. Phanikumar P, Stotz S C, Paramashivappa R, Beedle M, Zamponi G W and Srinivasa Rao A $2002 \mathrm{Mol}$. Pharmacol. 61649
9. Narayana Swamy B, Suma T K, Venkateswara Rao G and Chandrasekara Reddy G 2007 Eur. J. Med. Chem. 42422

10. Kubo I, Ochi M, Viera P C and Komatsu S 1993 J. Agric. Food Chem. 411012

11. Mantelingu K, Kishore A $\mathrm{H}$, Balasubramanyam K, Kumar G V, Altaf M, Swamy S N, Selvi R, Das C, Narayana C, Rangappa K S and Kundu T K 2007 J. Phys. Chem. B $\mathbf{1 1 1} 4527$

12. Hari Kishore A, Vedamurthy B M, Mantelingu K, Agrawal S, Ashok Reddy B A, Roy S, Rangappa K S and Kundu T K 2008 J. Med. Chem. 51792

13. Sung B, Pandey M K, Ahn K S, Yi T, Chaturvedi M M, Liu M and Aggarwal B 2008 Blood 1114880

14. Cui L, Miao J, Furuya T, Fan Q, Li X, Rathod P K, Su X Z and Cui L 2008 Eukaryotic Cell 71200

15. Sbardella G, Castellano S, Vicidomini C, Rotili D, Nebbioso A, Miceli M, Altucci L and Mai A 2008 Bioorg. Med. Chem. Lett. 182788

16. Rybak M J and Akins R L 2001 Drugs 61, 1

17. Lipsitch M 2001 Trends Microbiol. 9438

18. Cunha B A 1998 Drugs Today 34691

19. Marchese A, Schito G C and Debbia E A $2000 \mathrm{~J}$. Chemother., (Firenze) $\mathbf{1 2} 459$

20. Cetinkaya Y, Falk P and Mayhall C G 2000 Clin. Microbiol. Rev. 13686

21. Cassell G H and Mekalanos J $2001 \mathrm{~J}$. Am. Med. Assoc. 285601

22. Chu D T W, Plattner J J and Katz L 1996 J. Med. Chem. 393853

23. Gulati A S and Subba Rao B C 1964 Indian J. Chem. 2337

24. EI Sholy M A, Adawadkar P D, Benigni D A, Watson E S and Little T L Jr. 1986 J. Med. Chem. 29 606

25. (a) Dos Santos M L and de Magalhães G C 1993 Quim Nova, 16, 534; (b) Carvalho M G, Braz-Filho R, Dos Santos M L and de Magalhães G C 1993 J. Braz. Chem. Soc. 4 158; (c) Dos Santos M L and de Magalhães 1999 J. Braz. Chem. Soc. 10 13; (d) Lúcio P L Logrado, Dâmaris Silveira, Luiz A S Romeiro, Manoel O de Moraes, Bruno C Cavalcanti, Letícia V Costa-Lotufo, Cláudia do Ó Pessoa and Maria Lucilia dos Santos 2005 J. Braz. Chem. Soc. 161217

26. (a) Kiong L S and Tyman J H P 1981 J. Chem. Soc. Perkin Trans. 1 1942; (b) Paramashivappa R, Phanikumar P, Vithayathil P J and Srinivasa Rao A 2001 J. Agric. Food Chem. 492548

27. Rahman A, Choudhary M I and Thomsen W J 2001 Bioassay techniques for drug development (The Netherlands: Harwood Academic Publishers) pp. 16 